University of Nebraska - Lincoln

DigitalCommons@University of Nebraska - Lincoln

May 1989

\title{
Variational principle for high-order perturbations with application to multiphoton processes for the $\mathrm{H}$ atom
}

\author{
Bo Gao \\ University of Nebraska - Lincoln \\ Anthony F. Starace \\ University of Nebraska-Lincoln, astarace1@unl.edu
}

Follow this and additional works at: https://digitalcommons.unl.edu/physicsstarace

Part of the Physics Commons

Gao, Bo and Starace, Anthony F., "Variational principle for high-order perturbations with application to multiphoton processes for the H atom" (1989). Anthony F. Starace Publications. 37.

https://digitalcommons.unl.edu/physicsstarace/37

This Article is brought to you for free and open access by the Research Papers in Physics and Astronomy at DigitalCommons@University of Nebraska - Lincoln. It has been accepted for inclusion in Anthony F. Starace Publications by an authorized administrator of DigitalCommons@University of Nebraska - Lincoln. 


\title{
Variational principle for high-order perturbations with application to multiphoton processes for the $\mathrm{H}$ atom
}

\author{
Bo Gao and Anthony F. Starace \\ Department of Physics and Astronomy, The University of Nebraska, Lincoln, Nebraska 68588-0111
}

(Received 27 December 1988)

\begin{abstract}
The variationally stable procedure for $N$ th-order perturbative-transition-matrix elements introduced by the authors [Phys. Rev. Lett. 61, 404 (1988)] is presented here in detail. Its key features are that it is noniterative, involves only two unknown functions regardless of the value of $N$, is stable even near intermediate-state resonances (due to the presence of energy numerators rather than energy denominators), and uses the inverse of the perturbation operator for $N \geq 3$. Explicit formulas are presented for application to high-order multiphoton processes in atomic hydrogen. $\mathrm{Nu}$ merical results for multiphoton-ionization cross sections (for two, three, and seven photons), for the frequency dependence of the nonlinear susceptibilities for harmonic generation (for the third, fifth, and seventh harmonics), and for harmonic-generation transition rates up to 11 th order for $\lambda=1064$ $\mathrm{nm}$ are presented for the atomic hydrogen ground state and compared with results of others by more standard procedures. We also present detailed analyses of the $Z$ scaling of our results for hydrogenic systems, of the use of the imaginary part of the appropriate $2 \mathrm{~N}$-photon nonlinear susceptibility to obtain $N$-photon-ionization cross sections, and of the relation of the variational principle presented here to variational principles for scattering processes of Nuttall and Cohen [Phys. Rev. 188, 1542 (1969)] and of Schwinger [Phys. Rev. 72, 742 (1947)].
\end{abstract}

\section{INTRODUCTION}

The accuracy and utility of using variational principles for the calculation of quantities of physical interest are well known. ${ }^{1}$ While their use is common for scattering processes in order to obtain reliable approximations to the relevant transition-matrix amplitudes, ${ }^{2-4}$ their use is rare $^{5}$ for processes which are perturbative and of high order, as is often the case with multiphoton processes. For an $N$ th-order perturbative process, the major difficulty comes in summing over $N-1$ complete sets of intermediate states. Alternatively, use of the Dalgarno-Lewis procedure $^{6}$ reduces the problem formally to the solution of $N-1$ coupled inhomogeneous differential equations. For photon energies near intermediate-state resonances, the numerical accuracy of either method is often significantly reduced. In addition to these formal aspects, recent $a b$ initio calculations ${ }^{7,8}$ of two-photon-ionization cross sections for rare-gas atoms and their isoelectronic ions have shown that in such multielectron systems electroncorrelation effects play a major role in the multiphoton ionization process. Quantifying that role accurately for the high-order multiphoton processes studied experimentally is a major theoretical challenge. Clearly, then, there are many aspects of the theoretical description of $\mathrm{Nth}$ order perturbative processes (and of multiphoton processes in particular) which would benefit from a formulation that is designed to have variational stability.
We present in Sec. II a variationally stable formulation for an $N$ th-order perturbative process which addresses the major difficulty in evaluating higher-order amplitudes, namely, the summation over infinite numbers of intermediate states. Our expression for such amplitudes is variationally stationary with respect to deviations of the approximations to these summations from the exact results. Furthermore, our formulation avoids the introduction of energy denominators; hence, there are no singularities due to these denominators near intermediate-state resonance energies to affect the numerical accuracy of our results.

The key feature of our variational formulation for perturbative processes of third order and higher is the introduction of the inverse of the perturbation operator. This inverse operator requires a separate theoretical analysis for each perturbative process studied. Multiphoton processes for the hydrogen atom represent the general case of a one-electron perturbation operator acting on a oneelectron system. This case may be treated analytically. We do so for multiphoton processes for $\mathrm{H}$ and other hydrogenic systems in Sec. III. Applications of the formulation in Sec. III to the calculation of the second-, third-, and seventh-order multiphoton ionization cross sections, the linear susceptibility, the third-, fifth-, and seventhorder nonlinear susceptibilities, and the third-, fifth-, seventh-, ninth-, and 11th-order harmonic-generation transition rates in atomic $\mathrm{H}$ are presented in Sec. IV. 
In Sec. $\mathrm{V}$, we give a brief discussion of the relation of the present variational principle to well-known variational principles for scattering processes, ${ }^{2-4}$ and discuss other future applications of the present method. Finally, in Sec. VI we present some conclusions on the applications we have carried out so far for multiphoton processes. A brief description of this variational principle and its applications to two- and three-photon-ionization cross sections in atomic $\mathrm{H}$ has been given elsewhere. ${ }^{5}$

\section{VARIATIONALLY STABLE FORMULATION FOR AN $N$ th-ORDER PERTURBATIVE PROCESS}

\section{A. Standard procedures for $\mathbf{N}$ th-order perturbations}

Consider a perturbation operator $D$, which acts on some system and induces an $N$ th-order transition from an initial state $i$ to a final state $f$, which is not necessarily different from state $i$. The $N$ th-order transition amplitude may be written

$$
T_{i \rightarrow f}^{(N)}=\left\langle f\left|D \frac{1}{E(N-1)-H} D \cdots D \frac{1}{E(2)-H} D \frac{1}{E(1)-H} D\right| i\right\rangle,
$$

where the energies $E(n), 1 \leq n \leq N-1$, are appropriately chosen for the particular perturbation. Thus, for a timeindependent perturbation, $E(n)=E_{i}$, where $E_{i}$ is the zeroth-order energy of the initial state $i$. For a harmonic, time-dependent perturbation of frequency $\omega$, such as when $D$ represents the electric dipole operator as in multiphoton processes, $E(n)$ will generally depend on $\omega$. For example, if Eq. (1) represents the amplitude for an $N$ photon absorption process, then

$$
E(n)=E_{i}+n \omega .
$$

Equation (1) is, of course, written in operator form. $\mathrm{Nu}$ merical evaluation of Eq. (1) normally requires an explicit representation for the Green's-function operators. If expanded in terms of the complete set of eigenstates of the Hamiltonian $H$, for example, then

$$
\frac{1}{E(n)-H}=\sum_{j}|j\rangle \frac{1}{E(n)-E_{j}}\langle j| .
$$

Clearly, this method of evaluation of Eq. (1) results in $N-1$ generally infinite summations over intermediate states. When the exact solutions of $H$ are not used to represent the Green's-function operator, then numerical accuracy is often compromised.

An alternative procedure for evaluation of Eq. (1) is that of Dalgarno and Lewis. ${ }^{6,9}$ Briefly, one defines the following $N-1$ states:

$$
\begin{aligned}
& |\lambda(1)\rangle \equiv \frac{1}{E(1)-H} D|i\rangle, \\
& |\lambda(2)\rangle \equiv \frac{1}{E(2)-H} D|\lambda(1)\rangle, \\
& |\lambda(N-1)\rangle \equiv \frac{1}{E(N-1)-H} D|\lambda(N-2)\rangle .
\end{aligned}
$$

These states may be evaluated as the solutions of the following $N-1$ coupled, inhomogeneous Schrödinger-type equations (with suitable boundary conditions): ${ }^{9}$

$$
\begin{aligned}
& {[E(1)-H]|\lambda(1)\rangle=D|i\rangle,} \\
& {[E(2)-H]|\lambda(2)\rangle=D|\lambda(1)\rangle,} \\
& {[E(N-1)-H]|\lambda(N-1)\rangle=D|\lambda(N-2)\rangle .}
\end{aligned}
$$

Solution of Eqs. (5a)-(5c) sequentially to obtain the state $|\lambda(N-1)\rangle$ then permits one to calculate the transition amplitude in Eq. (1) as

$$
T_{i \rightarrow f}^{(N)} \equiv\langle f|D| \lambda(N-1)\rangle
$$

Clearly, the need for explicit summations over intermediate states is avoided in this method. However, since the solution of each equation, except the last, becomes the source term for the next equation, any errors (or approximations to the exact solutions) therefore propagate to higher equations. Additionally, in practice one finds that the accuracy of the solutions of the differential equations is reduced near any zeros of the operators $[E(n)-H]$ since the homogeneous solutions of the differential equation then must be dealt with.

\section{B. Variationally stable formulation}

In the Dalgarno-Lewis procedure, ${ }^{6}$ Eq. (6) is not the only form for the transition amplitude defined in Eq. (1). Note firstly that from Eq. (4), the state $|\lambda(N-1)\rangle$ may be defined as

$$
|\lambda(N-1)\rangle \equiv \frac{1}{E(N-1)-H} D \cdots D \frac{1}{E(2)-H} D \frac{1}{E(1)-H} D|i\rangle .
$$

In a similar way, we may alternatively define a state $\langle\mu(N-1)|$ as follows:

$$
\langle\mu(n-1)| \equiv\langle f| D \frac{1}{E(N-1)-H} D \cdots D \frac{1}{E(2)-H} D \frac{1}{E(1)-H} .
$$

Then just as the transition amplitude may be defined in terms of $|\lambda(N-1)\rangle$ as in Eq. (6), we may alternatively write it as 


$$
T_{i \rightarrow f}^{(N)} \equiv\langle\mu(N-1)|D| i\rangle .
$$

Additionally, if we formally manipulate Eq. (7) to obtain

$$
D|i\rangle=[E(1)-H] \frac{1}{D}[E(2)-H] \frac{1}{D} \cdots \frac{1}{D}[E(N-1)-H]|\lambda(N-1)\rangle,
$$

then its substitution into Eq. (9) yields a third expression for the transition amplitude:

$$
T_{i \rightarrow f}^{(N)} \equiv\left\langle\mu(N-1)\left|[E(1)-H] \frac{1}{D}[E(2)-H] \frac{1}{D} \cdots \frac{1}{D}[E(N-1)-H]\right| \lambda(N-1)\right\rangle .
$$

Finally, the three equivalent forms for the transition amplitude in Eqs. (6), (9), and (11) may be combined to obtain

$$
\begin{aligned}
T_{i \rightarrow f}^{(N)}= & \langle f|D| \lambda(N-1)\rangle+\langle\mu(N-1)|D| i\rangle \\
& -\left\langle\mu(N-1)\left|[E(1)-H] \frac{1}{D}[E(2)-H] \frac{1}{D} \cdots \frac{1}{D}[E(N-1)-H]\right| \lambda(N-1)\right\rangle .
\end{aligned}
$$

Equation 12 is variationally stable with respect to deviations of the states $|\lambda(N-1)\rangle$ and $\langle\mu(N-1)|$ from their exact expressions, denoted by $\left|\lambda_{\mathrm{ex}}(N-1)\right\rangle$ and $\left\langle\mu_{\mathrm{ex}}(N-1)\right|$. That is, if we define

$$
|\lambda(N-1)\rangle \equiv\left|\lambda_{\mathrm{ex}}(N-1)\right\rangle+|\delta \lambda(N-1)\rangle
$$

$$
\langle\mu(N-1)| \equiv\left\langle\mu_{\mathrm{ex}}(N-1)\right|+\langle\delta \mu(N-1)|,
$$

and substitute in Eq. (12), we obtain [upon use of Eq. (10) for $\left|\lambda_{\mathrm{ex}}(N-1)\right\rangle$ and of a similar equation for $\left\langle\mu_{\mathrm{ex}}(N-1)\right|$ that may be derived from Eq. (8)]

$$
T_{i \rightarrow f}^{(N)}=T_{i \rightarrow f}^{(N)}(\mathrm{ex})-\left\langle\delta \mu(N-1)\left|[E(1)-H] \frac{1}{D}[E(2)-H] \frac{1}{D} \cdots \frac{1}{D}[E(N-1)-H]\right| \delta \lambda(N-1)\right\rangle .
$$

Equation (14) shows clearly that the deviation of the transition amplitude from the exact result, $T_{i \rightarrow f}^{(N)}(\mathrm{ex})$, is of second order in $\delta \mu$ and $\delta \lambda$. In contrast, if Eq. (13a) is substituted in Eq. (6), then

$$
T_{i \rightarrow f}^{(N)}=T_{i \rightarrow f}^{(N)}(\mathrm{ex})+\langle f|D| \delta \lambda(N-1)\rangle,
$$

which shows a linear dependence on $\delta \lambda$.

\section{Key additional features of Eq. (12)}

Our variationally stable form for an $N$ th-order perturbative transition amplitude, Eq. (12), has several additional features which we highlight here. First, though it employs states of the Dalgarno-Lewis ${ }^{6}$ type, there is no iteration procedure as in the standard procedure described in Sec. II A above. Alternatively stated, our Eq. (12) makes use of only a pair of functions, $\mu(N-1)$ and $\lambda(N-1)$, independent of the order $N$ of the process.

Second, our Eq. (12) for the transition amplitude is stable near resonances. Indeed, in contrast to the standard perturbative expression for the transition amplitude in Eq. (1), our Eq. (12) has energy numerators instead of energy denominators.

Third, Eq. (12) in a sense makes the evaluation of $N$ thorder perturbative transition amplitudes equivalent in effort to that for a first-order transition amplitude. Namely, for any order $N$, attention is focused on the initial and final states, $i$ and $f$, which are the experimentally observed states. Expansions of the two unknown functions, $\lambda(N-1)$ and $\mu(N-1)$, in terms of some basis, with determination of the expansion coefficients by application of the principle of variational stability, replace the standard task of summing $N-1$ intermediate sets of states.

Fourth, evaluation of the third form for the transition amplitude on the right-hand side of Eq. (12) is in general nontrivial due to the appearance of the inverse of the perturbation operator. How this is accomplished in the case of perturbative-multiphoton processes in atomic hydrogen is described in Sec. III A.

\section{VARIATIONALLY STABLE FORMULATION FOR PERTURBATIVE MULTIPHOTON PROCESSES IN HYDROGENIC SYSTEMS}

\section{A. Formulation for atomic $\mathbf{H}$}

For a one-electron system such as atomic $\mathbf{H}$, the numerical evaluation of the variationally stable form for the transition amplitude, Eq. (12), is straightforward. The key is to use Eq. (12) to evaluate only the radial part of the standard form for the transition amplitude, Eq. (1). The angular part of the amplitude is evaluated analytically for Eq. (1) by standard techniques, which require no discussion here. Indeed, for multiphoton-ionization processes in atomic $\mathrm{H}$, formulas for the relevant angular factors have been presented by Gontier and Trahin. ${ }^{9}$ Using their notation, ${ }^{9}$ the radial part of the standard form for the multiphoton transition amplitude, Eq. (1), for a transition from a hydrogenic initial state $\left|n_{i} l_{i}\right\rangle$ to a hydrogenic final state $\left|n_{f} l_{f}\right\rangle$ by $N$ polarized photons of frequency $\omega$ is 


$$
P\left(l_{1} l_{2}, \ldots, l_{N-1}, l_{f} \mid \omega\right)=\left\langle n_{f} l_{f}\left|r \frac{1}{E(N-1)-h\left(l_{n-1}\right)} r \cdots r \frac{1}{E(2)-h\left(l_{2}\right)} r \frac{1}{E(1)-h\left(l_{1}\right)} r\right| n_{i} l_{i}\right\rangle .
$$

As discussed below Eq. (1), $E(n)$, where $1 \leq n \leq N-1$, must be chosen appropriately for the process under consideration. For a lowest-order, $N$-photon absorption or ionization process, Eq. (2) applies. The notation $\left|n_{i} l_{i}\right\rangle$ and $\left\langle n_{f} l_{f}\right|$ denotes only the radial parts of the initial and final hydrogenic states. The radial part of the transition operator $D$ in the electric dipole approximation is simply $r$; all effects due to the polarization of the photons are treated analytically in calculating the angular part of Eq. (1). The set $l_{1}, l_{2}, \ldots, l_{N-1}$ represents one particular set of intermediate-state orbital angular momenta. The total
$N$-photon transition amplitude is expressed in terms of a summation over all allowed sets of such angular momenta. ${ }^{9}$ Finally, the one-electron radial Hamiltonian for a particular orbital angular momentum $l$ is defined by

$$
h(l) \equiv-\frac{1}{2} d^{2} / d r^{2}-1 / r+l(l+1) / 2 r^{2} .
$$

The derivation of a variationally stable expression for the radial transition amplitude in Eq. (16) proceeds as in Sec. II above. In place of Eqs. (7) and (8), one defines now their radial equivalents, $\lambda_{r}$ and $\mu_{r}$,

$$
\begin{aligned}
& \left|\lambda_{r}(N-1)\right\rangle \equiv \frac{1}{E(N-1)-h\left(l_{N-1}\right)} r \cdots r \frac{1}{E(2)-h\left(l_{2}\right)} r \frac{1}{E(1)-h\left(l_{1}\right)} r\left|n_{i} l_{i}\right\rangle, \\
& \left\langle\mu_{r}(N-1)\right| \equiv\left\langle n_{f} l_{f}\right| r \frac{1}{E(N-1)-h\left(l_{N-1}\right)} r \cdots r \frac{1}{E(2)-h\left(l_{2}\right)} r \frac{1}{E(1)-h\left(l_{1}\right)} .
\end{aligned}
$$

The variationally stable expression for the radial amplitude in Eq. (16) is then

$$
\begin{aligned}
P\left(l_{1}, l_{2}, \ldots, l_{N-1}, l_{f} \mid \omega\right)= & \left\langle n_{f} l_{f}|r| \lambda_{r}(N-1)\right\rangle+\left\langle\mu_{r}(N-1)|r| n_{i} l_{i}\right\rangle \\
& -\left\langle\mu_{r}(N-1)\left|\left[E(1)-h\left(l_{1}\right)\right] \frac{1}{r}\left[E(2)-h\left(l_{2}\right)\right] \frac{1}{r} \cdots \frac{1}{r}\left[E(N-1)-h\left(l_{N-1}\right)\right]\right| \lambda_{r}(N-1)\right\rangle,
\end{aligned}
$$

which is the radial analogue of the general result in Eq. (12).

\section{B. Z scaling for hydrogenic ions}

Any result for an $N$-photon transition amplitude for atomic hydrogen may be easily scaled for application to a hydrogenic ion of nuclear charge $Z$. One merely takes note of the following scaling relations for the Hamiltonian, the radial Green's function, and for bound and continuum states, respectively,

$$
\begin{aligned}
& H_{Z}(r)=Z^{2} H_{\mathrm{H}}(Z r), \\
& \frac{1}{E_{i}(Z)+q \omega-H_{Z}(r)} \\
& \quad=\frac{1}{Z^{2}} \frac{1}{E_{i}(Z=1)+q\left(\omega / Z^{2}\right)-H_{\mathrm{H}}(Z r)}, \\
& |n l ; r\rangle_{Z}=Z^{3 / 2}|n l ; Z r\rangle_{\mathrm{H}}, \\
& |E l ; r\rangle_{Z}=Z^{1 / 2}\left|\left(E / Z^{2}\right) l ; Z r\right\rangle_{\mathrm{H}} .
\end{aligned}
$$

In Eq. (21) the subscript $Z(\mathbf{H})$ on the kets and on the Hamiltonian indicates that these correspond to a hydrogenic system with ionic charge $Z$ (atomic $\mathbf{H}$ ), $n$ and $l$ indicate the principal and orbital angular momentum quantum numbers for a bound state, $E l$ represents the electron's kinetic energy and orbital angular momentum for a continuum state, $q$ represents an integer, and $\omega$ is the photon frequency.

These properties give the following scaling relations for bound-bound, bound-continuum, and continuum-continuum $N$-photon perturbative transition amplitudes for hydrogenic ions, respectively:

$$
\begin{aligned}
& { }_{Z}\left\langle n^{\prime} l^{\prime}\left|T_{Z}^{(N)}(\omega)\right| n l\right\rangle_{Z} \\
& =Z^{-(3 N-2)}{ }_{\mathrm{H}}\left\langle n^{\prime} l^{\prime}\left|T_{\mathrm{H}}^{(N)}\left(\omega / Z^{2}\right)\right| n l\right\rangle_{\mathrm{H}} \\
& { }_{Z}\left\langle E^{\prime} l^{\prime}\left|T_{Z}^{(N)}(\omega)\right| n l\right\rangle_{Z} \\
& =Z^{-(3 N-1)}{ }_{\mathrm{H}}\left\langle\left(E^{\prime} / Z^{2}\right) l^{\prime}\left|T_{\mathrm{H}}^{(N)}\left(\omega / Z^{2}\right)\right| n l\right\rangle_{\mathrm{H}}
\end{aligned}
$$

$$
\begin{aligned}
& { }_{Z}\left\langle E^{\prime} l^{\prime}\left|T_{Z}^{(N)}(\omega)\right| E l\right\rangle_{Z} \\
& \quad=Z^{-3 N_{\mathrm{H}}}\left\langle\left(E^{\prime} / Z^{2}\right) l^{\prime}\left|T_{\mathrm{H}}^{(N)}\left(\omega / Z^{2}\right)\right|\left(E / Z^{2}\right) l\right\rangle_{\mathrm{H}}
\end{aligned}
$$

In Eq. (22), $T^{(N)}$ is the operator defined in Eq. (1) and the argument in parentheses is the frequency of the photon in a multiphoton process.

We note that in obtaining the results in Eq. (22) from the scaling relationships in Eq. (21), it is necessary to change variables from $r$ to $t$ where $t=Z r$. This is necessary in order that the radial integrals on the right-hand 
side of Eq. (22) are independent of $Z$ except for the scale factor shown outside the matrix elements and the change in energy from $\omega$ and $E$ on the left-hand side of Eq. (22) to $\left(\omega / Z^{2}\right)$ and $\left(E / Z^{2}\right)$ on the right.

\section{RESULTS FOR PERTURBATIVE MULTIPHOTON PROCESSES IN ATOMIC H}

We present here a number of results for multiphoton ionization, for nonlinear susceptibilities for harmonic generation, and for harmonic-generation transition rates in atomic hydrogen to demonstrate the ability of the variationally stable formulation presented in Secs. II and III above to provide accurate numerical predictions. Where possible we have compared our results with predictions of others by the more standard techniques described in Sec. II. The agreement between our results and those of these more standard procedures is generally excellent. We also check our variationally stable procedure for selfconsistency by comparing the results of two alternative calculations for generalized multiphoton cross sections. The results of the two calculational methods agree very well.

\section{A. Numerical method}

For each radial transition amplitude in Eq. (20) to be evaluated, we expand the unknown functions $\lambda_{r}(N-1)$ and $\mu_{r}(N-1)$ in Slater orbitals, as follows:

$$
\begin{aligned}
& \lambda_{r}(N-1)(r)=\sum_{j=1}^{M} a_{j} \phi_{j}(r), \\
& \mu_{r}(N-1)(r)=\sum_{j=1}^{M} b_{j} \theta_{j}(r),
\end{aligned}
$$

where the Slater orbitals are defined by

$$
\begin{aligned}
& \phi_{j}(r)=N_{j} r^{l_{N-1}+j} e^{-\beta r}, \\
& \theta_{j}(r)=N_{j}^{\prime} r^{l_{1}+j} e^{-\beta r},
\end{aligned}
$$

where $N_{j}$ and $N_{j}^{\prime}$ are normalization constants and where $\beta$ is an arbitrary constant which is chosen heuristically for each calculation and which may be complex. Occasionally, functions having different values of $\beta$ are employed in the expansions in Eq. (23). In many cases, however, a single value for $\beta$ suffices and hence, for simplicity of notation, we shall only consider this case explicitly.

Substitution of Eq. (23) into Eq. (20) gives an expression for $P\left(l_{1}, l_{2}, \ldots, l_{N-1}, l_{f} \mid \omega\right)$ in terms of radial matrix elements for Slater orbitals, which may be evaluated analytically. Requiring variational stability of $P$ with respect to the expansion coefficients $a_{j}$ and $b_{j}$, i.e., setting for each $j$,

$$
\frac{\partial P}{\partial a_{j}}=\frac{\partial P}{\partial b_{j}}=0
$$

one obtains the following set of equations for the expansion coefficients:

$$
\begin{aligned}
& \sum_{j=1}^{M} A_{i j} a_{j}=c_{i}, \\
& \sum_{i=1}^{M} b_{i} A_{i j}=d_{j},
\end{aligned}
$$

where the radial matrix elements involving the Slater orbitals (and also the initial and final radial wave functions, $\left\langle r \mid n_{i} l_{i}\right\rangle$ and $\left.\left\langle r \mid n_{f} l_{f}\right\rangle\right)$ have been defined by

$$
\begin{aligned}
& A_{i j}=\left\langle\theta_{i}\left|\left[E(1)-h\left(l_{1}\right)\right] \frac{1}{r}\left[E(2)-h\left(l_{2}\right)\right] \frac{1}{r} \cdots \frac{1}{r}\left[E(N-1)-h\left(l_{N-1}\right)\right]\right| \phi_{j}\right\rangle, \\
& c_{i} \equiv\left\langle\theta_{i}|r| n_{i} l_{i}\right\rangle \\
& d_{j} \equiv\left\langle n_{f} l_{f}|r| \phi_{j}\right\rangle .
\end{aligned}
$$

For the fourth through 12th-order processes presented below, the sizes of our calculations may be described as follows. In calculating the nonlinear susceptibilities, $M \approx 40$ and only a single value for $\beta$ is employed. For the seven-photon partial ionization cross section, we employed four values of $\beta$, each having an expansion involving $M \approx 30$ functions. In all cases our results converged to at least four significant digits, as tested by increasing the number of functions $\boldsymbol{M}$. This excellent convergence was obtained even near intermediate-state resonances (although a significant deterioration was found for intermediate energies falling into the high-Rydberg region or in the continuum).
Lastly we note that the major practical impediment to calculations of $N$ th-order perturbative matrix elements by this method, as by any other method, is simply the increase in the number of pathways [and hence in the number of radial amplitudes in Eq. (20)] by which the transition proceeds from the initial to the final state as $N$ increases.

\section{B. Results for multiphoton ionization of $\mathrm{H}(1 s)$}

The generalized cross section ${ }^{10}$ for multiphoton ionization of a state $|i\rangle$ in atomic hydrogen by $N$ photons leading to a final state $\langle f|$ may be written

$$
\sigma^{(N)} /\left(I^{N-1}\right)=\left(2^{3-N} \pi^{2} \alpha \omega\left(2 l_{i}+1\right)^{-1} \sum_{l_{f}, m_{f}, m_{i}}\left|T_{i \rightarrow f}^{(N)}\right|^{2}\right]\left[\frac{a_{0}^{2}}{I_{0}^{N-1}}\right]
$$


The generalized cross section on the left-hand side of Eq. (28), which has units of $\mathrm{cm}^{2 N} / \mathrm{W}^{N-1}$, is given by an expression on the right-hand side which is independent of the light intensity $I$. The ordinary cross section, denoted $\sigma^{(N)}$, has units of $\mathrm{cm}^{2}$ and depends on $I$ in lowest-order perturbation theory through the factor $I^{N-1}$, where $I$ is in units of $\mathrm{W} / \mathrm{cm}^{2}$. On the right-hand side, $\alpha$ is the finestructure constant, $\omega$ is the photon frequency, $l_{i}\left(l_{f}\right)$ and $m_{i}\left(m_{f}\right)$ are the electron's initial (final) -state orbital angular momentum and magnetic quantum numbers. The amplitude $T_{i \rightarrow f}^{(N)}$ has been defined generally in Eq. (1), where $D$ is the electric dipole operator for a particular light polarization. All of our calculations are done in atomic units $\left(e=\hbar=m_{e}=1\right)$ using the expression in the first set of parentheses on the right-hand side in Eq. (28). To convert to the units $\mathrm{cm}^{2 N} / \mathrm{W}^{N-1}$ we employ the conversion factor in the second set of parentheses on the right-hand side in Eq. (28) where $a_{0}$, the atomic unit of length, is the Bohr radius in centimeters, and where $I_{0}$, the atomic unit of intensity, is equal to $7.019 \times 10^{16}$ $\mathrm{W} / \mathrm{cm}^{2}$.

We have given extensive comparisons of our predictions for two- and three-photon ionization cross sections for $\mathbf{H}(1 s)$ with results of other authors in Ref. 5. In those comparisons, ${ }^{5}$ we agree consistently with predictions of Karule $^{11}$ for two-photon ionization, both above and below the single-photon ionization threshold. In order to test the accuracy of our variationally stable method of calculation for much higher-order processes, therefore, we again make comparisons with results of Karule, ${ }^{12}$ which are conveniently presented in tabular form. Karule's results ${ }^{12}$ are obtained by employing a Sturmian function expansion for the Green's functions in the transition operator in Eq. (1).

In Table I we compare Karule's results ${ }^{12}$ with ours for the seven-photon ionization of $\mathrm{H}(1 s)$ to the final state in which the photoelectron has orbital angular momentum $l_{f}=7$. Also given in Table $I$ is an indication of the convergence of our results. For the ten wavelengths shown for $\lambda \geq 5638 \AA$, our results either agree identically with Karule's or differ by considerably less than $1 \%$. For the four shorter wavelengths shown, the differences are somewhat greater, but still range between $0.6 \%$ and $2.2 \%$, which is very good agreement. We conclude therefore that our variational procedure is applicable with high accuracy to high orders of perturbation well beyond the second and third order, for which it was originally presented. ${ }^{5}$
TABLE I. Partial seven-photon ionization cross section $\left(\mathrm{cm}^{14} / \mathrm{W}^{6}\right)$ of $\mathrm{H}(1 s)$ for $l_{f}=7$ for linearly polarized light.

\begin{tabular}{lccc}
\hline \hline$\lambda(\AA)$ & $\begin{array}{c}\text { Sturmian } \\
\text { expansion method }\end{array}$ & $\begin{array}{c}\text { Present } \\
\text { variational method }\end{array}$ & $\begin{array}{c}\text { Convergence } \\
\text { factor }^{\mathrm{b}}\end{array}$ \\
\hline 6368 & $5.16 \times 10^{-107}$ & $5.120 \times 10^{-107}$ & $1.0 \times 10^{-9}$ \\
6205 & $3.45 \times 10^{-106}$ & $3.463 \times 10^{-106}$ & $7.7 \times 10^{-10}$ \\
6107 & $7.02 \times 10^{-106}$ & $7.032 \times 10^{-106}$ & $2.5 \times 10^{-8}$ \\
6020 & $1.13 \times 10^{-105}$ & $1.137 \times 10^{-105}$ & $1.5 \times 10^{-7}$ \\
5925 & $1.72 \times 10^{-105}$ & $1.717 \times 10^{-105}$ & $1.0 \times 10^{-6}$ \\
5796 & $2.62 \times 10^{-105}$ & $2.622 \times 10^{-105}$ & $1.9 \times 10^{-6}$ \\
5739 & $3.04 \times 10^{-105}$ & $3.044 \times 10^{-105}$ & $4.7 \times 10^{-6}$ \\
5716 & $3.22 \times 10^{-105}$ & $3.213 \times 10^{-105}$ & $6.4 \times 10^{-6}$ \\
5676 & $3.51 \times 10^{-105}$ & $3.509 \times 10^{-105}$ & $4.4 \times 10^{-6}$ \\
5658 & $3.64 \times 10^{-105}$ & $3.641 \times 10^{-105}$ & $1.1 \times 10^{-5}$ \\
5638 & $3.78 \times 10^{-105}$ & $3.789 \times 10^{-105}$ & $1.1 \times 10^{-5}$ \\
5612 & $3.93 \times 10^{-105}$ & $3.981 \times 10^{-105}$ & $3.8 \times 10^{-5}$ \\
5602 & $3.99 \times 10^{-105}$ & $4.059 \times 10^{-105}$ & $1.8 \times 10^{-4}$ \\
5590 & $4.13 \times 10^{-105}$ & $4.156 \times 10^{-105}$ & $5.1 \times 10^{-5}$ \\
5574 & $4.34 \times 10^{-105}$ & $4.246 \times 10^{-105}$ & $1.9 \times 10^{-4}$ \\
\hline \hline
\end{tabular}

${ }^{\mathrm{a}}$ E. Karule, Ref. 12.

${ }^{\mathrm{b}}$ Factor by which present-variational-method results change upon increasing the size of our basis from $4 \times 28$ to $4 \times 30$.

\section{Variationally stable complex-basis-expansion method for multiphoton ionization of $\mathrm{H}(1 s)$}

It is well known that the single-photon ionization cross section is proportional to the imaginary part of the frequency-dependent linear susceptibility. ${ }^{13}$ This fact has been employed recently, together with complex-basisexpansion methods, to calculate total photoionization cross sections. ${ }^{14}$ Less well known, perhaps, is the fact that similar relations hold between the $N$-photon ionization cross sections and the imaginary parts of the corresponding frequency-dependent $2 \mathrm{~N}$-photon nonlinear susceptibilities. ${ }^{15}$ As a test of the consistency of our variationally stable method, we calculate two- and threephoton ionization cross sections for $\mathrm{H}(1 s)$ by this alternative method for comparison with our results employing Eq. (28), which have been presented in Ref. 5.

The relation between the generalized cross section ${ }^{10}$ for multiphoton ionization by $N$ photons below the $N-1$ photon ionization threshold and the $2 N$-photon nonlinear susceptibility is

$$
\left.\sigma^{(N)} /\left(I^{N-1}\right)=2^{3-N} \pi \alpha \omega\left(2 l_{i}+1\right)^{-1} \sum_{m_{i}} \operatorname{Im}\left|\left\langle i\left|\left(T^{(N)}\right)^{\dagger} \frac{1}{E_{i}+N \omega-H} T^{(N)}\right| i\right\rangle\right| \mid \frac{a_{0}^{2}}{I_{0}^{N-1}}\right) .
$$

Nearly all the quantities in Eq. (29) are the same as in Eq. (28) except that the absolute square of the transition amplitude and the sum over final states in Eq. (28) is replaced in Eq. (29) by the imaginary part of the nonlinear susceptibility appropriate for absorption of $N$ photons followed by emission of $N$ photons in which the energy
$\left[E_{i}+(N-1) \omega\right]$ is below the $N-1$-photon ionization threshold. In Eq. (29) the operator $T^{(N)}$ denotes the operator inside the matrix element on the right-hand side of Eq. (1). Clearly, a calculation using Eq. (29) for the nonlinear susceptibility is of twice the order of a calculation employing Eq. (28), in which one calculates the tran- 
sition amplitude. Thus our results by our variationally stable method applied to Eq. (29) for $N=2$ and 3 are of fourth and sixth order, respectively, as compared to our results for the same processes using Eq. (28), which are of second and third order.

Comparisons of the two procedures are presented in Table II for $N=2$ and in Table III for $N=3$. One sees in each case that for the longest wavelengths agreement between the two alternative procedures is excellent. For the shorter wavelengths, however, the accuracy of the complex-basis-expansion method deteriorates. (We know that our transition amplitude calculations are reliable from the comparisons with others presented in Ref. 5.) We understand this deterioration in accuracy of the complex-basis-expansion method as due primarily to the increasing energy above threshold of the $N$ th photon and not due to the higher order of the calculations. Representation of the continuum by discrete basis expansion techniques is known to be more difficult than the corresponding representation of bound states.

\section{Nonlinear susceptibilities for harmonic generation of light from $\mathrm{H}(1 s)$}

The nonlinear process of harmonic generation by the ground state of atomic hydrogen may be described by an expansion of the induced polarization in odd powers of the incident electric field, ${ }^{15,16}$ where each term in this expansion may be written

$$
P^{(q)}=2^{1-q} \chi^{(q)}(\omega) E^{q} .
$$

Here $P^{(q)}$ is the $q$ th-order polarization, $\chi^{(q)}(\omega)$ is the $q$ thorder nonlinear susceptibility for producing the harmonic of frequency $q \omega$ when the incident electromagnetic wave has frequency $\omega, E$ is the incident electric field, and $2^{1-q}$ is a normalization coefficient.

In general, many processes contribute to each $\chi^{(q)}$ and hence it is not possible to present a general expression for $\chi^{(q)}$. For the third-order $(q=3)$ nonlinear susceptibility, Ward ${ }^{17}$ gives diagrams for all four terms which contribute in his Table VI. All of our calculations are carried out in atomic units $\left(e=m_{e}=\hbar=1\right)$ and then converted to the more common esu units, as follows:

$$
\chi^{(q)}(\mathrm{esu})=\left(a_{0}^{2 q+1} / e^{q-1}\right) \chi^{(q)}(\text { a.u. }) .
$$

TABLE II. Variational calculation of the two-photon ionization cross section $\left(\mathrm{cm}^{4} / \mathrm{W}\right)$ of $\mathrm{H}(1 \mathrm{~s})$ for linearly polarized light.

\begin{tabular}{ccc}
\hline$\lambda(\AA)$ & $\begin{array}{c}\text { Transition } \\
\text { amplitude } \\
\text { calculation }^{\mathrm{a}}\end{array}$ & $\begin{array}{c}\text { Complex-basis } \\
\text { expansion method }\end{array}$ \\
\hline 1700 & $1.025 \times 10^{-32}$ & $1.025 \times 10^{-32}$ \\
1600 & $9.153 \times 10^{-33}$ & $9.162 \times 10^{-33}$ \\
1400 & $8.451 \times 10^{-33}$ & $8.473 \times 10^{-33}$ \\
1300 & $1.276 \times 10^{-32}$ & $1.290 \times 10^{-32}$ \\
1200 & $6.441 \times 10^{-32}$ & $7.134 \times 10^{-32}$ \\
\hline \hline
\end{tabular}

${ }^{a}$ Reference 5. This is a second-order calculation employing Eq. (28).

${ }^{\mathrm{b}}$ Fourth-order calculation employing Eq. (29).
TABLE III. Variational calculation of the three-photon ionization cross section $\left(\mathrm{cm}^{6} / \mathbf{W}^{2}\right)$ of $\mathbf{H}(1 s)$ for linearly polarized light.

\begin{tabular}{lcc}
\hline \hline & $\begin{array}{c}\text { Transition } \\
\text { amplitude } \\
\text { calculation }^{\mathrm{a}}\end{array}$ & $\begin{array}{c}\text { Complex-basis } \\
\text { expansion method }\end{array}$ \\
\hline 2600 & $1.002 \times 10^{-46}$ & $1.010 \times 10^{-46}$ \\
2500 & $2.948 \times 10^{-46}$ & $2.914 \times 10^{-46}$ \\
2400 & $7.057 \times 10^{-46}$ & $7.366 \times 10^{-46}$ \\
2300 & $2.641 \times 10^{-47}$ & $2.235 \times 10^{-47}$ \\
\hline \hline
\end{tabular}

${ }^{a}$ Reference 5. This is a third-order calculation employing Eq. (28).

${ }^{\mathrm{b}}$ Sixth-order calculation employing Eq. (29).

Here $a_{0}$ is the Bohr radius and $e$ is the electron charge.

Our results for the frequency dependence of $\chi^{(q)}(\omega)$ for $q=1,3,5$, and 7 are presented in Figs. 1-4. For $q=1$, of course, $\chi^{(1)}$ is the usual linear susceptibility. In each of the figures, the resonance behavior arising from coincidence of $E_{1 s}+m \omega$ ( $m=$ integer) with intermediate-state energy levels $E_{n}$ of hydrogen is clearly seen. In fact, when such coincidences occur simultaneously in an even number of intermediate states, the nonlinear susceptibility fails to change its sign at the resonance, as occurs in Fig. 3 near $\lambda=4860 \AA$ at which there is a five-photon resonance of the ground state with the $n=4$ level as well as a four-photon resonance of the ground state and the $n=2$ level. This coincidence, of course, only occurs in hydrogen.

Another interesting feature of the susceptibilities shown in Figs. 1-4 is whether or not they change sign between resonances. For example, in Fig. 1 for the linear susceptibility, there is always a change in sign between resonances as the photon energy increases and $E_{1 s}+\omega$ passes from just greater than $E_{n}$ to just less than $E_{n+1}$ for $n=2,3, \ldots$ In Figs. 2-4, however, above the $n=2$ resonance with $E_{1 s}+q \omega$, the susceptibilities $\chi^{(q)}(\omega)$ stay

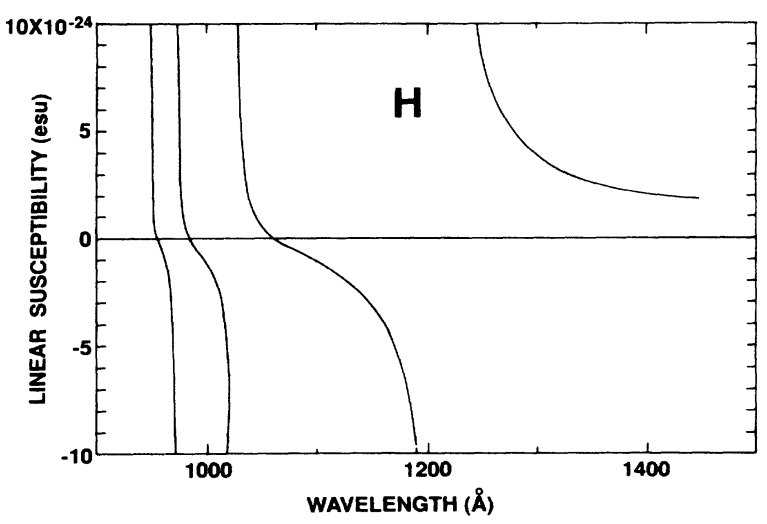

FIG. 1. Linear susceptibility (in esu) of atomic hydrogen for wavelengths $900 \AA \leq \lambda \leq 1500 \AA$. 


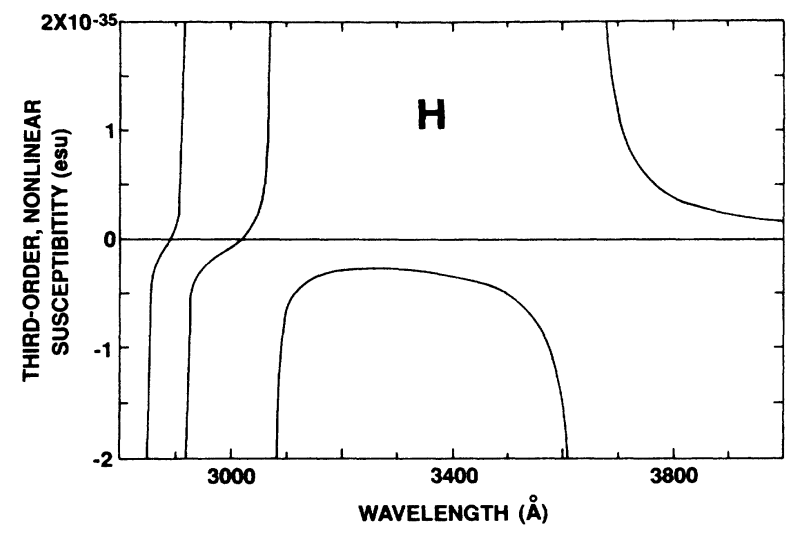

FIG. 2. Third-order nonlinear susceptibility (in esu) of atomic hydrogen for wavelengths $2800 \AA \leq \lambda \leq 4000 \AA$.

negative until above the $n=3$ resonance with $E_{1 s}+q \omega$. These behaviors may be understood by a rather detailed examination, which we do not present here, of the dominant contributions to $\chi^{(q)}$ near each of the resonances. One should note for the purpose of this examination that the radial matrix elements for electric dipole transitions in atomic hydrogen are known to be positive definite as long as initial and final states have different principal quantum numbers. ${ }^{18}$ On the other hand, these radial matrix elements are negative definite, with value ${ }^{19}$

$$
\langle n l \pm 1|r| n l\rangle=-\frac{3}{2} n\left(n^{2}-l_{>}^{2}\right)^{1 / 2},
$$

when the principal quantum numbers are the same.

\section{E. Transition rates for harmonic generation of light from $H(1 s)$ for $\lambda=1064 \mathrm{~nm}$}

The transition rate (in units of $\mathrm{sec}^{-1}$ ) for harmonic generation of wavelength $\lambda_{0} / q$ induced by incident radiation of wavelength $\lambda_{0}$ is

$$
W^{(q)}=(3 \hbar)^{-1}\left(2 \pi q / \lambda_{0}\right)^{3}\left|P^{(q)}\right|^{2} .
$$

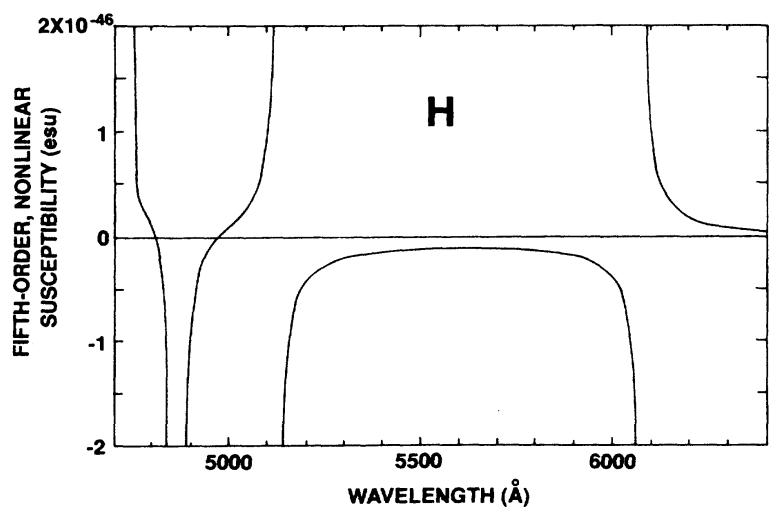

FIG. 3. Fifth-order nonlinear susceptibility (in esu) of atomic hydrogen for wavelengths $4700 \AA \leq \lambda \leq 6400 \AA$.

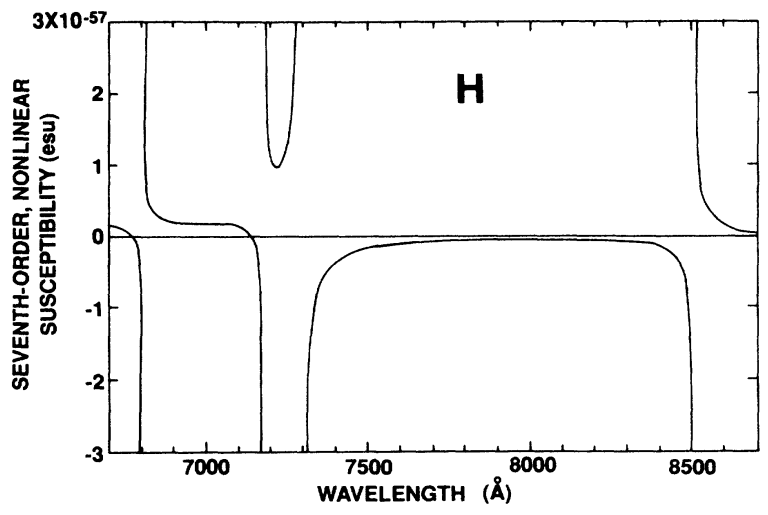

FIG. 4. Seventh-order nonlinear susceptibility (in esu) of atomic hydrogen for wavelengths $6700 \AA \leq \lambda \leq 8700 \AA$.

When $P(q)$ is substituted from Eq. (30) and $E^{2}$ is written in terms of the intensity $I$ of the incident light,

$$
E^{2}=8 \pi I / c
$$

then $W^{(q)}$ may be expressed in terms of the nonlinear susceptibility as

$$
W^{(q)}=(4 / 3 h)\left(2 \pi q / \lambda_{0}\right)^{3}(2 \pi I / c)^{q}\left|\chi^{(q)}\right|^{2} .
$$

In Table IV we compare our predictions for $W^{(q)}$ for incident light of wavelength $\lambda_{0}=1064 \mathrm{~nm}$ and for incident intensity $I=3 \times 10^{13} \mathrm{~W} / \mathrm{cm}^{2}=3 \times 10^{20}$ esu with results of Potvliege and Shakeshaft. ${ }^{20}$ These authors have used standard perturbation methods together with $q$ Sturmian basis expansions (one for each of the Green's functions that occur in the $q$ th-order nonlinear susceptibility). Our calculations for $\chi^{(q)}$ employ two Slater-orbital expansions of about 40 functions each to evaluate the appropriate variationally stable radial transition amplitudes in Eq. (20). We see that for $3 \leq q \leq 11$, our results agree to within $0.026 \%$ or better with the infinite nuclear mass results of Potvliege and Shakeshaft. ${ }^{20}$

In order to show the trend of harmonic-generation transition rates with both intensity and harmonic order, we show in Fig. 5 a plot of $\log _{10}\left(R^{(q)}\right)$, where the ratio $R^{(q)}$ is defined by

$$
\begin{aligned}
R^{(q)} \equiv & W^{(q)} / W^{(3)} \\
= & (q / 3)^{3}[2 \pi I(\text { esu }) / c(\text { esu })]^{q-3} \\
& \times\left[\chi^{(q)}(\text { esu }) / \chi^{(3)}(\text { esu })\right]^{2} \\
= & \left.(q / 3)^{3}[I \text { (a.u. }) / 4\right]^{q-3} \\
& \times\left[\chi^{(q)}(\text { a.u. }) / \chi^{(3)}(\text { a.u. })\right]^{2}
\end{aligned}
$$

We make this plot for comparison with experimental measurements of Ferray et al. ${ }^{21}$ for the relative intensity of harmonics (compared to the third harmonic) generated in the rare gases. One sees in Fig. 5 that the steep drop in the ratio $R^{(q)}$ for $q=5$ as compared to $q=3$ is in agreement with the experiments, which were carried out for an 
TABLE IV. Transition rate $\left(\mathrm{sec}^{-1}\right)$ for harmonic generation in atomic $\mathbf{H}\left[q \gamma+\mathbf{H}(1 s) \rightarrow \gamma^{\prime}+\mathbf{H}(1 s)\right]$ for a laser wavelength $\lambda=1064 \mathrm{~nm}$ and laser intensity $I=3 \times 10^{13} \mathrm{~W} / \mathrm{cm}^{2}$.

\begin{tabular}{cccc}
\hline \hline & \multicolumn{2}{c}{ Sturmian expansion method $^{\mathrm{a}}$} \\
$\begin{array}{c}\text { Harmonic } \\
\text { order } q\end{array}$ & $\begin{array}{c}\text { Finite nuclear } \\
\text { mass }^{\mathrm{a}}\end{array}$ & $\begin{array}{c}\text { Infinite nuclear } \\
\text { mass }^{\mathrm{b}}\end{array}$ & $\begin{array}{c}\text { Present } \\
\text { variational } \\
\text { method }\end{array}$ \\
\hline 3 & $3.46 \times 10^{1}$ & $3.4273 \times 10^{1}$ & $3.428 \times 10^{1}$ \\
5 & $7.78 \times 10^{-1}$ & $7.6614 \times 10^{-1}$ & $7.662 \times 10^{-1}$ \\
7 & $5.35 \times 10^{-1}$ & $5.2092 \times 10^{-1}$ & $5.210 \times 10^{-1}$ \\
9 & $5.78 \times 10^{2}$ & $5.7687 \times 10^{2}$ & $5.770 \times 10^{2}$ \\
11 & $5.21 \times 10^{5}$ & $6.5063 \times 10^{5}$ & $6.508 \times 10^{5}$ \\
\hline \hline
\end{tabular}

${ }^{a}$ Reference 20(a).

${ }^{\mathrm{b}}$ Reference 20(b).

intensity $I=3 \times 10^{13} \mathrm{~W} / \mathrm{cm}^{2}$. The plateau found experimentally in the rare gases for $q \geq 7$ is found in our calculations for atomic hydrogen at significantly lower intensities. We caution, however, that use of lowest-order perturbation theory becomes unreliable whenever $R^{(q+2)}$ $\approx R^{(q)}$ since effects of order $q+2$ are neglected in calculating $R^{(q)}$. Nevertheless, the tendency for $R^{(q)}$ to flatten out for $q \geq 7$ as $I$ increases is clearly shown in our perturbative results for $I=0.5 \times 10^{12} \mathrm{~W} / \mathrm{cm}^{2}$, for which lowest-order perturbation theory barely holds. For this intensity, $W^{(5)} / W^{(7)}=5.29 \times 10^{3}$ but $W^{(7)} / W^{(9)}=3.25$.

\section{DISCUSSION}

\section{A. Relation to variational principles for scattering processes}

The variational principle for the $N$ th-order perturbation matrix element which we have introduced in Ref. 5 and presented in detail in this paper reduces for $N=2$

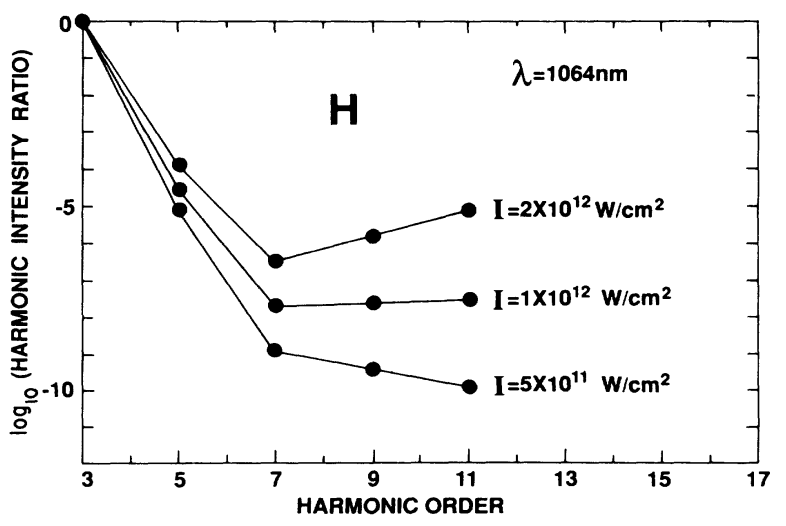

FIG. 5. Logarithmic harmonic-generation transition-rate ratio, $\log _{10}\left(\mathrm{~W}^{(q)} / \mathrm{W}^{(3)}\right)$, plotted vs harmonic order $q$ for $3 \leq q \leq 11$ for wavelength $\lambda=1064 \mathrm{~nm}$. Results for three laser intensities are shown. The relation of the transition rate ratio $R^{(q)} \equiv \mathbf{W}^{(q)} / \mathbf{W}^{(3)}$ to the nonlinear susceptibilities is given in $\mathbf{E q}$. (36) of the text. (i.e., for second-order processes) to a form analogous to well-known variational principles for scattering processes. We investigate this relationship here. Of course, the variational principles for scattering processes apply to the exact transition-matrix elements, whereas above we have been concerned with the $N$ th-order perturbative approximation to the exact transition-matrix element.

We adopt in this section without detailed explanation the well-known notation of Lippmann and Schwinger ${ }^{2(b)}$ for the transition matrix $T_{b a}$ between scattering states $a$ and $b$,

$$
T_{b a}=\left(\psi_{b}^{(-)}\left|H_{1}\right| \Phi_{a}\right)=\left(\Phi_{b}\left|H_{1}\right| \psi_{a}^{(+)}\right),
$$

where

$$
\psi_{a(b)}^{( \pm)}=\Phi_{a(b)}+\frac{1}{E \pm i \epsilon-H_{0}} H_{1} \psi_{a(b)}^{( \pm)} .
$$

Here the $\psi$ 's are exact solutions to the full Hamilton $H \equiv H_{0}+H_{1}$, while the $\Phi$ 's are solutions of the zerothorder Hamiltonian $H_{0}$. Each scattering solution corresponds to the energy $E$, and the notation \pm as well as the infinitesimal $\pm i \epsilon$ indicate outgoing or incoming wave boundary conditions.

In analogy with Sec. II of this paper, define now formally

$$
\begin{aligned}
\lambda_{a(b)}^{( \pm)} & \equiv \psi_{a(b)}^{( \pm)}-\Phi_{a(b)} \\
& =\frac{1}{E \pm i \epsilon-H_{0}} H_{1} \psi_{a(b)}^{( \pm)} .
\end{aligned}
$$

Equation (40) implies that

$$
\left(E-H_{0}\right) \lambda_{a(b)}^{( \pm)}+H_{1} \psi_{a(b)}^{( \pm)} \text {. }
$$

We now derive several alternative forms for $T_{b a}$ which may be combined to yield a variationally stable form for $T_{b a}$ with respect to changes in the functions $\lambda_{a}^{( \pm)}$and $\lambda_{b}^{( \pm)}$. Firstly, using Eq. (39) to substitute for $\psi_{a}^{(+)}$in the second expression for $T_{b a}$ in Eq. (37), we obtain

$$
T_{b a}=t_{b a}+\left(\Phi_{b}\left|H_{1}\right| \lambda_{a}^{(+)}\right),
$$

where $t_{b a}$ is the zeroth-order transition-matrix element $\left(\Phi_{b}\left|H_{1}\right| \Phi_{a}\right)$. Similarly, using Eq. (39) to substitute for 
$\psi_{b}^{(-)}$in the first expression for $T_{b a}$ in Eq. (37) gives

$$
T_{b a}=t_{b a}+\left(\lambda_{b}^{(-)}\left|H_{1}\right| \Phi_{a}\right) .
$$

Lastly, Eqs. (39) and (41) allow us to write

$$
\left(\lambda_{b}^{(-)}\left|H_{1}\right| \Phi_{a}\right)=\left(\lambda_{b}^{(-)}|(E-H)| \lambda_{a}^{(+)}\right) .
$$

Combining Eqs. (42)-(44) we may write a variationally stable form $T_{b a}^{\prime}$ for the transition amplitude, as follows:

$$
\begin{aligned}
T_{b a}^{\prime}= & t_{b a}+\left(\Phi_{b}\left|H_{1}\right| \lambda_{a}^{(+)}\right)+\left(\lambda_{b}^{(-)}\left|H_{1}\right| \Phi_{a}\right) \\
& -\lambda_{b}^{(-)}|(E-H)| \lambda_{a}^{(+)} .
\end{aligned}
$$

Equation (45) has been obtained by Nuttall and Cohen ${ }^{22}$ and is analogous to our Eq. (12) for the special case of a second-order process (i.e., $N=2$ ). As in our calculations, $\lambda_{b}^{(-)}$and $\lambda_{a}^{(+)}$may be expanded in some basis and the expansion coefficients determined by insisting that Eq. (45) be variationally stable. This form for $T_{b a}^{\prime}$ has all the key features of our Eq. (12) that are described in Sec. II C above.

Equation (45) may be rewritten in the form of the Schwinger variational principle for $T_{b a}$ by using Eqs. (39) $-(41)$ to replace $\lambda_{a}^{(+)}$and $\lambda_{b}^{(-)}$. Thus, from Eq. (39), the first three terms in Eq. (45) may be written as

$$
\begin{aligned}
t_{b a}+\left(\Phi_{b}\left|H_{1}\right| \lambda_{a}^{(+)}\right)+\left(\lambda_{b}^{(-)}\left|H_{1}\right| \Phi_{a}\right) \\
=-t_{b a}+\left(\Phi_{b}\left|H_{1}\right| \psi_{a}^{(+)}\right)+\left(\psi_{a}^{(-)}\left|H_{1}\right| \Phi_{a}\right) .
\end{aligned}
$$

The last term in Eq. (45) may be written

$$
\begin{aligned}
\lambda_{b}^{(-)}|(E-H)| & \lambda_{a}^{(+)} \\
= & \left(\lambda_{b}^{(-)}\left|E-H_{0}\right| \lambda_{a}^{(+)}\right)-\left(\lambda_{a}^{(-)}\left|H_{1}\right| \lambda_{a}^{(+)}\right) .
\end{aligned}
$$

The first term on the right-hand side of Eq. (47) may be rewritten by using Eq. (39) to replace $\lambda_{b}^{(-)}$and Eq. (41) to replace $\left(E-H_{0}\right) \lambda_{a}^{(+)}$. The second term on the righthand side of Eq. (47) may be rewritten by using Eq. (40) to replace $\lambda_{a}^{(+)}$and Eq. (39) to replace $\lambda_{b}^{(-)}$. Collecting these results and using Eq. (38) we obtain

$$
\begin{aligned}
\lambda_{b}^{(-)} \mid & (E-H) \mid \lambda_{a}^{(+)} \\
= & \left(\psi_{b}^{(-)}\left|H_{1}\right| \psi_{a}^{(+)}\right)-t_{b a} \\
& -\left\langle\psi_{b}^{(-)}\left|H_{1} \frac{1}{E+i \epsilon-H_{0}} H_{1}\right| \psi_{a}^{(+)}\right\rangle .
\end{aligned}
$$

Substituting Eqs. (46) and (48) into Eq. (45) we obtain the Schwinger ${ }^{23}$ variational principle for $T_{b a}$

$$
\begin{aligned}
T_{b a}^{\prime}= & \left(\psi_{b}^{(-)}\left|H_{1}\right| \Phi_{a}\right)+\left(\Phi_{b}\left|H_{1}\right| \psi_{a}^{(+)}\right)-\left(\psi_{b}^{(-)}\left|H_{1}\right| \psi_{a}^{(+)}\right) \\
& +\left(\psi_{b}^{(-)}\left|H_{1} \frac{1}{E+i \epsilon-H_{0}} H_{1}\right| \psi_{a}^{(+)}\right)
\end{aligned}
$$

Comparing Eqs. (45) and (49) we see that Eq. (49) has four terms containing the (unknown) exact wave functions $\psi_{a(b)}^{( \pm)}$as compared to only three terms involving the (unknown) exact $\lambda$ 's in Eq. (45). Furthermore, Eq. (49) involves the matrix element of an energy denominator as compared to the matrix element of an energy numerator in Eq. (45). These differences may have practical computational consequences.

\section{B. Other applications of the variationally stable procedure for $\boldsymbol{N}$ th-order perturbative processes}

The key aspect of any extension of the variationally stable procedure for $N$ th-order perturbative processes presented in Sec. II above is the treatment of the inverse of the perturbation operator. The treatment shown for multiphoton processes in atomic hydrogen would apply to any other perturbation in atomic hydrogen, as, for example, due to external electric or magnetic fields. Extending the treatment for such one-body perturbation operators to nonhydrogenic systems is straightforward as long as single-electron excitations out of some groundstate configuration are the only excitations considered. If multiconfiguration basis states involving possibly two or more excited electrons are introduced, then care must be taken to evaluate the inverse operator for each set of configuration types. Also, for a nonhydrogenic, manyelectron system, it may not be feasible to evaluate the integrand in the last term in Eq. (12) analytically, even in some approximate way, in which case the numerical aspects of this evaluation become a concern. Certainly, however, Eq. (12) may be evaluated in second order $(N=2)$ for any perturbation operator in any system, since then the last term reduces essentially to a matrix element of the full Hamiltonian $H$.

Another extension of our variationally stable procedure is to the calculation of perturbative amplitudes for excitations well into the continuum. As illustrated in Sec. IV C above, this requires the use of complex basis functions and a larger number of basis functions. However, Gontier et al. ${ }^{24}$ and Potvliege and Shakeshaft ${ }^{25}$ have achieved good convergence of their results by standard procedures for high orders of perturbation theory well above the ionization threshold. We anticipate, therefore, no major obstacles to the use of our variational method in this region.

\section{CONCLUSIONS}

We have given in this paper a detailed presentation of a variationally stable procedure for $N$ th-order perturbative-transition-matrix elements that was introduced in Ref. 5. Among the key features of this procedure are the absence of $N$ th-order iterations, the simplicity of having only two unknown functions regardless of the value of $N$, the stability of the procedure even near intermediate-state resonances (due to the absence of energy denominators), and the introduction of the inverse of the transition operator. Detailed expressions for use of this procedure for high-order multiphoton processes in atomic hydrogen have been presented. The accuracy of this method has been demonstrated by calculated results for multiphoton cross sections, nonlinear susceptibilities, and harmonicgeneration transition rates, which have been shown to be in excellent agreement with results of more standard cal- 
culations. We have also presented detailed analyses of the $Z$ scaling of our results for hydrogenic systems, of the use of the imaginary part of the appropriate nonlinear susceptibility (together with complex basis functions) to obtain multiphoton ionization cross sections, and of the relation of the variational principle presented here for $N$ th-order perturbative processes to variational principles for scattering processes of Nuttall and Cohen ${ }^{22}$ and Schwinger. ${ }^{2,23}$

\section{ACKNOWLEDGMENTS}

This work was supported in part by National Science Foundation Grant No. PHY-8601429.
${ }^{1}$ E. Gerjuoy, A. R. P. Rau, and L. Spruch, Rev. Mod. Phys. 55, 725 (1983).

2(a) J. Schwinger, Phys. Rev. 72, 742 (1947); (b) B. A. Lippmann and J. Schwinger, ibid. 79, 469 (1950).

${ }^{3}$ Roger G. Newton, Scattering Theory of Waves and Particles (McGraw-Hill, New York, 1966), Sec. 11.3.

${ }^{4}$ J. Nuttall and H. L. Cohen, Phys. Rev. 188, 1542 (1969).

${ }^{5}$ B. Gao and A. F. Starace, Phys. Rev. Lett. 61, 404 (1988).

${ }^{6}$ A. Dalgarno and J. T. Lewis, Proc. R. Soc. London, Ser. A 233, 70 (1955).

${ }^{7}$ A. L'Huillier, L. Jönsson, and G. Wendin, Phys. Rev. A 33, 3938 (1986); A. L'Huillier and G. Wendin, J. Phys. B 20, L37 (1987); 21, L247 (1988).

${ }^{8}$ A. F. Starace and T. F. Jiang, Phys. Rev. A 36, 1705 (1987); T. F. Jiang and A. F. Starace, ibid. 38, 2347 (1988).

${ }^{9}$ Y. Gontier and M. Trahin, Phys. Rev. 172, 83 (1968).

${ }^{10}$ P. Lambropoulos, Adv. At. Mol. Phys. 12, 87 (1976).

${ }^{11}$ E. Karule, J. Phys. B 11, 441 (1978).

${ }^{12}$ E. Karule, in Atomic Processes (Zinatne, Riga, USSR, 1975), pp. 5-24 (in Russian).

${ }^{13}$ U. Fano and J. W. Cooper, Rev. Mod. Phys. 40, 441 (1968), Secs. 2.1 and 2.2 .

${ }^{14}$ T. N. Rescigno, Phys. Rev. A 31, 607 (1985); T. N. Rescigno and C. W. McCurdy, ibid. 31, 624 (1985).
${ }^{15} \mathrm{R}$. Loudon, The Quantum Theory of Light (Clarendon, Oxford, 1973), pp. 307-312.

${ }^{16}$ N. B. Delone and V. P. Krainov, Atoms in Strong Light Fields, Vol. 28 of Springer Series in Chemical Physics, edited by V. I. Goldanskii, R. Gomer, F. P. Schäfer, and J. P. Toennies (Springer, Berlin, 1985), Sec. 7.1.

${ }^{17}$ J. F. Ward, Rev. Mod. Phys. 37, 1 (1965).

${ }^{18}$ S. D. Oh and R. H. Pratt, Phys. Rev. A 34, 2486 (1986).

${ }^{19}$ H. A. Bethe and E. E. Salpeter, Quantum Mechanics of Oneand Two-Electron Atoms (Springer, Berlin, 1957), Eq. (63.5). Note that this equation has a misprint: The right-hand side should be multiplied by -1 .

${ }^{20}$ (a) R. M. Potvliege and R. Shakeshaft, Z. Phys. D 11, 93 (1989); (b) R. M. Potvliege (private communication).

${ }^{21}$ M. Ferray, A. L'Huillier, X. F. Li, L. A. Lompre, G. Mainfray, and C. Manus, J. Phys. B 21, L31 (1988).

22J. Nutall and H. L. Cohen, Phys. Rev. 188, 1542 (1969), cf. Eq. (4).

${ }^{23}$ Cf. Ref. 2(b), Eq. (1.78); and Ref. 3, Eq. (11.68).

${ }^{24}$ Y. Gontier, N. K. Rahman, and M. Trahin, Phys. Rev. A 34, 1112 (1986).

${ }^{25}$ R. M. Potvliege and R. Shakeshaft, Phys. Rev. A 39, 1545 (1989). 